\title{
Transoral robotic thyroidectomy using the da Vinci single-port surgical system
}

\author{
Kyung Tae \\ Department of Otolaryngology-Head and Neck Surgery, College of Medicine, Hanyang University, Seoul, Republic of Korea \\ Correspondence to: Kyung Tae, MD, PhD. Department of Otolaryngology-Head and Neck Surgery, College of Medicine, Hanyang University, 222 \\ Wangsimni-ro, Seongdong-gu, Seoul 04763, Republic of Korea. Email: kytae@hanyang.ac.kr. \\ Provenance and Peer Review: This article was commissioned by the editorial office, Gland Surgery. The article did not undergo external peer review. \\ Comment on: Chan JYK, Koh YW, Richmon J, et al. Transoral thyroidectomy with a next generation flexible robotic system: A feasibility study in a \\ cadaveric model. Gland Surg 2019;8:644-7.
}

Submitted Feb 25, 2020. Accepted for publication Mar 10, 2020.

doi: $10.21037 / g s .2020 .03 .37$

View this article at: http://dx.doi.org/10.21037/gs.2020.03.37

Of the various remote access thyroidectomy techniques using a surgical robot or endoscopy, transoral approach has been recently popularized worldwide owing to its advantages of less morbidity and good cosmesis (1). The surgical invasiveness related to the creation of the working space is least in the transoral approach as compared with the bilateral axillo-breast approach (BABA) and transaxillary approaches. Postoperative cosmesis might be better than other remote access procedures because it leaves no visible skin scar. In addition, an advantage of the transoral approach is that it enables total thyroidectomy and complete central neck dissection, even up to level VII, because it is a midline approach and the surgical view is superior to the inferior direction (1).

A surgical robot is commonly used rather than the endoscopic procedure in the transaxillary, BABA, and postauricular facelift approaches, although the robotic procedure is costly. The endoscopic procedure has several limitations such as the two-dimensional surgical view, restrictions and collisions in instrument manipulations, and the fulcrum effect in that the tool end points move in the direction opposite to the surgeon's hands due to the pivot point $(1,2)$. In addition, it needs assistants to hold the endoscope. Therefore, robotic thyroidectomy using the da Vinci Si or Xi surgical system (Intuitive Surgical Inc., Sunnyvale, CA, USA) has been introduced to overcome the limitations of endoscopic procedures. Robotic procedures provide a three-dimensional magnified view, fine motion scaling, hand-tremor filtering, innovative instrumentation with extended freedom of motion, and surgical education (1-3).

However, in the transoral approach, despite the many advantages of the robotic procedure, the endoscopic procedure via the vestibular approach is more commonly adopted worldwide than the robotic procedure. This might be related to the high cost of the robotic procedure, and the availability and need for training on the surgical robot system. The robotic procedure is costlier than the endoscopic procedure and is not available in all institutes. Moreover, practice is required for learning the technique for using the surgical robot system and being familiar with the procedure to avoid complications and overcome the learning curve. A small additional axillary skin incision is necessary to place the third robotic instrument. The robotic procedure takes more time than the endoscopic procedure in transoral thyroidectomy $(2,3)$.

However, transoral robotic thyroidectomy using the da Vinci Si or Xi system has many advantages than the transoral endoscopic procedure $(2,3)$. In addition to the main advantages of the da Vinci surgical system, such as the three-dimensional magnified view and innovative instrumentation with extended freedom of motion, the ability to use three robotic instruments simultaneously with the axillary port is especially crucial for obtaining counter traction, which facilitates dissection and improves surgical dexterity and ergonomics with the robotic procedure. Counter traction leads to a safer and more precise dissection as in the conventional thyroidectomy, especially for dissection and preservation of the parathyroid glands and recurrent laryngeal nerve. With counter traction of the 
tissue, the dissection of the upper pole can be performed more conveniently and easily. Moreover, external stitches for the retraction of the strap muscle and the skin flap are not necessary in the robotic procedure if a third robotic instrument is available. Also, it is convenient to take out the large surgical specimens through the axillary port.

Recently, Chan et al. reported the technique of transoral robotic thyroidectomy with the da Vinci single port (SP) surgical system (Intuitive Surgical Inc.) and $\mathrm{CO}_{2}$ insufflation in a cadaveric model (4). In addition, Park et al. reported a similar technique of transoral robotic thyroidectomy using the da Vinci SP surgical system in two human cadavers (5). They made an approximately $2-\mathrm{cm}$ incision in the central oral vestibule and two 5-mm lateral incisions, and elevated the skin flap using laparoscopic endoscopy and instruments. After creating the working space, the central oral incision was extended to approximately $2.5-3 \mathrm{~cm}$ to deploy the da Vinci SP robot, and the two lateral incisions were sutured. The single-port cannula tip of the da Vinci SP surgical system was suspended in the air approximately $6-10 \mathrm{~cm}$ apart from the oral mucosal incision. A plastic wound retractor was used to connect the cannula tip of the SP system and the oral mucosal incision for sealing off the working space and insufflating $\mathrm{CO}_{2}$ gas. One endoscope and three robotic instruments in the single cannula of the SP system were deployed for thyroidectomy.

The da Vinci SP system might provide some advantages than the da Vinci Si or Xi system in transoral thyroid surgery. In the single-port cannula of the SP system, all four robotic extensions, including a bendable tip camera and three multi-joint robotic instruments can be placed through a central oral incision without an additional axillary trocar. Therefore, we can avoid the axillary skin scar from the third robotic instrument. However, some limitations and disadvantages remain. The size of the single-port cannula of the SP system is $2.5 \mathrm{~cm}$. Therefore, the central oral incision should be $>2.5-3 \mathrm{~cm}$ to place the singleport cannula in the air and deploy the all three robotic instruments and one endoscope through the central oral incision. The large central oral incision of at least $>2.5-3 \mathrm{~cm}$ tended to damage the mental nerve. Even if there is no direct nerve damage, the mental nerve damage can increase by increased pressure to the nerve by the large oral incision for deploying all robotic instruments and endoscope of the SP system. In addition, to deploy the robotic instruments in the working space, the tip of cannula of the SP system should be suspended in the air. Therefore, the cannula tip and oral mucosal incision must be taped for sealing off the space to insufflate the $\mathrm{CO}_{2}$ gas by using the plastic wound retractor. However, it might not be easy and convenient to maintain the sealing-off status using a plastic wound retractor through the operation. Energy-based devices are effective in dividing the blood vessels and securing hemostasis in thyroid surgery. However, the energy devices, such as Harmonic curved shears are not available in the da Vinci SP system. One of the important advantage of the robotic procedure than the endoscopic procedure in transoral thyroidectomy is its ability to perform counter traction using the three robotic instruments. However, the effectiveness of counter traction using the three robotic instruments in the SP system may be limited because the distance between the instruments is close in the SP system. Therefore, counter traction of the thyroid and surrounding tissue effectively might not be enough as compared with the da Vinci Si or Xi system.

Furthermore, recently, Park et al. reported the technique of gasless transoral robotic thyroidectomy using the da Vinci SP system in human patients (6). In their technique, they elevated the skin flap through a $3-\mathrm{cm}$ central oral vestibular incision under direct vision. The external retractor system was used to maintain the working space without $\mathrm{CO}_{2}$ insufflations. They placed an endoscope and only two robotic instruments in the single cannula of the SP system through the central oral incision. They could not place the third robotic instrument in the SP cannula of the SP system because the central oral incision port is not enough to place all robotic instruments and the external retractor for the working space. The gasless method has the advantage of the absence of complications related to carbon dioxide insufflation (7). However, gasless transoral robotic thyroidectomy using the da Vinci SP system does not have the most important advantage of robotic surgery, which can use the three robotic instruments for the counter traction. In addition, maintaining the working space with the external retractor is not as good as the $\mathrm{CO}_{2}$ insufflations method. It requires more soft tissue dissection in the chin area to create the working space under direct vision and to make enough space to place the external skin retractor and robotic system. It might result in a high possibility of mental nerve injury and soft tissue damage. Therefore, the gasless transoral robotic thyroidectomy technique using the da Vinci SP system does not seem to be better than the technique with $\mathrm{CO}_{2}$ insufflations.

In conclusion, transoral robotic thyroidectomy with the da Vinci SP surgical system and $\mathrm{CO}_{2}$ insufflation is feasible. However, considering its various limitations, the 
da Vinci SP surgical system does not seem to offer major advantages over the da Vinci Si or Xi surgical system in transoral thyroidectomy. To overcome these limitations, we need a new surgical robot that is smaller, more flexible, and equipped with an energy device.

\section{Acknowledgments}

Funding: None.

\section{Footnote}

Conflicts of Interest: The author has completed the ICMJE uniform disclosure form (available at http://dx.doi. org/10.21037/gs.2020.03.37). The author has no conflicts of interest to declare.

Ethical Statement: The author is accountable for all aspects of the work in ensuring that questions related to the accuracy or integrity of any part of the work are appropriately investigated and resolved.

Open Access Statement: This is an Open Access article distributed in accordance with the Creative Commons Attribution-NonCommercial-NoDerivs 4.0 International License (CC BY-NC-ND 4.0), which permits the noncommercial replication and distribution of the article with the strict proviso that no changes or edits are made and the original work is properly cited (including links to both the formal publication through the relevant DOI and the license). See: https://creativecommons.org/licenses/by-nc-nd/4.0/.

\section{References}

1. Tae K, Ji YB, Song CM, et al. Robotic and Endoscopic Thyroid Surgery: Evolution and Advances. Clin Exp Otorhinolaryngol 2019;12:1-11.

2. Tae K, Lee DW, Song CM, et al. Early experience of transoral thyroidectomy: Comparison of robotic and endoscopic procedures. Head Neck 2019;41:730-8.

3. Tae K, Ji YB, Song CM, et al. Safety and efficacy of transoral robotic and endoscopic thyroidectomy: The first 100 cases. Head Neck 2020;42:321-9.

4. Chan JYK, Koh YW, Richmon J, et al. Transoral thyroidectomy with a next generation flexible robotic system: A feasibility study in a cadaveric model. Gland Surg 2019;8:644-7.

5. Park D, Shaear M, Chen YH, et al. Transoral robotic thyroidectomy on two human cadavers using the Intuitive da Vinci single port robotic surgical system and $\mathrm{CO} 2$ insufflation: Preclinical feasibility study. Head Neck 2019;41:4229-33.

6. Park YM, Kim DH, Moon YM, et al. Gasless transoral robotic thyroidectomy using the $\mathrm{DaVinci}$ SP system: feasibility, safety, and operative technique. Oral Oncol 2019;95:136-42.

7. Kim KN, Lee DW, Kim JY, et al. Carbon dioxide embolism during transoral robotic thyroidectomy: A case report. Head Neck 2018;40:E25-8.
Cite this article as: Tae $\mathrm{K}$. Transoral robotic thyroidectomy using the da Vinci single-port surgical system. Gland Surg 2020;9(3):614-616. doi: 10.21037/gs.2020.03.37 